\title{
Reproducing kernel functions for the generalized Kuramoto-Sivashinsky equation
}

\author{
Ali Akgül ${ }^{1, *}$, Esra Karatas Akgül ${ }^{2, * *}$, Sahin Korhan $^{3, * * *}$, and Mustafa Inc ${ }^{4, * * * *}$ \\ ${ }^{1}$ Siirt University, Art and Science Faculty, Department of Mathematics, 56100, Siirt/TURKEY \\ ${ }^{2}$ Siirt University, Faculty of Education, Department of Mathematics, 56100, Siirt/TURKEY \\ ${ }^{3}$ Siirt University, Art and Science Faculty, Department of Mathematics, 56100, Siirt/TURKEY \\ ${ }^{4}$ Firat University, Faculty of Science, Department of Mathematics, 23119, Elazig/TURKEY
}

Abstract. Reproducing kernel functions are obtained for the solution of generalized Kuramoto-Sivashinsky (GKS) equation in this paper. These reproducing kernel functions are valuable in the reproducing kernel Hilbert space method. They will be useful for interested researchers.

\section{Introduction}

We consider the following problem[1]:

$$
\frac{\partial v}{\partial t}+v \frac{\partial v}{\partial x}+\alpha \frac{\partial^{2} v}{\partial x^{2}}+\beta \frac{\partial^{3} v}{\partial x^{3}}+\gamma \frac{\partial^{4} v}{\partial x^{4}}=0
$$

where $\alpha, \beta$ and $\gamma$ are nonzero [2]. This equation has been obtained in the context of plasma instabilities, flame front propagation, and phase turbulence in reaction-diffusion system [3]. We obtain reproducing kernel functions to get approximate solutions of the following problem:

Example In this example, we consider the Kuramoto-Sivashinsky equation [5]

$$
v_{t}+v v_{x}+v_{x x}+v_{x x x x}=0,
$$

with the initial condition [5]

$$
v(x, 0)=\exp \left(-x^{2}\right)
$$

and the boundary conditions

$$
v(a, t)=0, \quad v(b, t)=0, \quad v_{x}(a, t)=0, \quad v_{x}(b, t)=0 .
$$

\footnotetext{
*e-mail: aliakgu100727@gmail.com

**e-mail: esrakaratas@siirt.edu.tr

***e-mail: sahin_3192@hotmail.com

****e-mail: minc@ firat.edu.tr
} 


\section{Reproducing kernel functions}

We define $V_{2}^{5}[0,1]$ as

$$
V_{2}^{5}[0,1]=\left\{\begin{array}{cc}
v, v^{\prime}, v^{\prime \prime}, v^{\prime \prime \prime}, v^{(4)} & \text { are absolutely continuous functions, } \\
v^{(5)} \in L^{2}[0,1], & v(a)=v^{\prime}(a)=v(b)=v^{\prime}(b)=0 .
\end{array}\right\}
$$

We give

$$
\begin{aligned}
\left\langle v, S_{z}\right\rangle_{V_{2}^{5}[a, b]} & =v(a) S_{z}(a)+v^{\prime}(a) S_{z}^{\prime}(a)+v^{\prime \prime}(a) S_{z}^{\prime \prime}(a)+v^{\prime \prime \prime}(a) S_{z}^{\prime \prime \prime}(a) \\
& +v^{(4)}(a) S_{z}^{(4)}(a)+\int_{a}^{b} v^{(5)}(t) S_{z}^{(5)}(t) d(t)
\end{aligned}
$$

We obtain

$$
\begin{aligned}
\left\langle v, S_{z}\right\rangle_{V_{2}^{5}[a, b]} & =v(a) S_{z}(a)+v^{\prime}(a) S_{z}^{\prime}(a)+v^{\prime \prime}(a) S_{z}^{\prime \prime}(a)+u^{\prime \prime \prime}(a) S_{z}^{\prime \prime \prime}(a) \\
& +v^{(4)}(a) S_{z}^{(4)}(a)+v^{(4)}(b) S_{z}^{(5)}(b)-v^{(4)}(a) S_{z}^{(5)}(a) \\
& -v^{(3)}(b) S_{z}^{(6)}(b)+v^{(3)}(a) S_{z}^{(6)}(a) \\
& +v^{\prime \prime}(b) S_{z}^{(7)}(b)-v^{\prime \prime}(a) S_{z}^{(7)}(a)-v^{\prime}(b) S_{z}^{(8)}(b) \\
& +v^{\prime}(a) S_{z}^{(8)}(a)+v(b) S_{z}^{(9)}(b)-v(a) S_{z}^{(9)}(a) \\
& -\int_{a}^{b} v(t) S_{z}^{(10)}(t) d(t) .
\end{aligned}
$$

We have

$$
\begin{aligned}
& S_{z}(a)=0 \\
& S_{z}(b)=0 \\
& S_{z}^{\prime}(a)=0 \\
& S_{z}^{\prime}(b)=0
\end{aligned}
$$

by boundary conditions. Therefore, we obtain

$$
\begin{aligned}
\left\langle v, S_{z}\right\rangle_{V_{2}^{5}[a, b]} & =v^{\prime \prime}(a) S_{z}^{\prime \prime}(a)+v^{\prime \prime \prime}(a) S_{z}^{\prime \prime \prime}(a)+v^{(4)}(a) S_{z}^{(4)}(a) \\
& +v^{(4)}(b) S_{z}^{(5)}(b)-v^{(4)}(a) S_{z}^{(5)}(a)-v^{(3)}(b) S_{z}^{(6)}(b)+v^{(3)}(a) S_{z}^{(6)}(a) \\
& +v^{\prime \prime}(b) S_{z}^{(7)}(b)-v^{\prime \prime}(a) S_{z}^{(7)}(a)-\int_{a}^{b} v(t) S_{z}^{(10)}(t) d(t)
\end{aligned}
$$


We have

$$
\begin{aligned}
S_{z}^{\prime \prime}(a)-S_{z}^{(7)}(a) & =0 \\
S_{z}^{\prime \prime \prime}(a)+S_{z}^{(6)}(a) & =0 \\
S_{z}^{(4)}(a)-S_{z}^{(5)}(a) & =0 \\
S_{z}^{(5)}(b) & =0 \\
S_{z}^{(6)}(b) & =0 \\
S_{z}^{(7)}(b) & =0
\end{aligned}
$$

Therefore, we obtain

$$
\left\langle v, S_{z}\right\rangle_{V_{2}^{5}[a, b]}=-\int_{a}^{b} v(t) S_{z}^{(10)}(t) d(t)=v(z) .
$$

Thus, we get

$$
S_{z}^{(10)}(t)=-\delta(t-z)
$$

When $\quad t \neq z$

$$
S_{z}^{(10)}(z)=0
$$

Then, we acquire

$$
S_{z}(t)= \begin{cases}\sum_{i=1}^{10} c_{i}(z) t^{i-1}, & t \leq z \\ \sum_{i=1}^{10} d_{i}(z) t^{i-1} & , \quad t>z .\end{cases}
$$

\section{Conclusion}

In this paper, we obtained very useful reproducing kernel function for solving the generalized Kuramoto-Sivashinsky equation. This reproducing kernel function is very useful to apply reproducing kernel Hilbert space method. 


\section{References}

[1] A.H. Khater, R.S. Temsah, Numerical solutions of the generalized Kuramoto-Sivashinsky equation by Chebyshev spectral collocation methods, Comput. Math. Appl. 56 1465-1472 (2008).

[2] Y. Kuramoto, T. Tsuzuki, Persistent propagation of concentration waves in dissipative media far from thermal equilibrium, Prog. Theor. Phys. 55 356-369 (1976).

[3] J. Rademacher J, R. Wattenberg, Viscous shocks in the destabilized Kuramoto-Sivashinsky, J. Comput. Nonlinear Dynam. 1 336-347 (2006).

[4] R. Conte R, Exact solutions of nonlinear partial differential equations by singularity analysis, in: Lecture Notes in Physics, Springer, Berlin, 2003, pp. 1-83.

[5] M. Lakestani, M. Dehghan, Numerical solutions of the generalized KuramotoSivashinsky equation using B-spline functions, Applied Mathematical Modelling 36 605-617 (2012).

[6] F. Genga, F. Shenb, Solving a Volterra integral equation with weakly singular kernel in the reproducing kernel space, Mathematical Sciences, Vol. 4, No. 2 159-170 (2010). 